EUROPEAN JOURNAL OF PURE AND APPLIED MATHEMATICS

Vol. 15, No. 1, 2022, 15-29

ISSN 1307-5543 - ejpam.com

Published by New York Business Global

\title{
Supra $b$ limit points and supra $b$ separation axioms
}

\author{
Tareq M. Al-shami ${ }^{1}$, Abdelwaheb Mhemdi ${ }^{2, *}$, Mohammed Jameel ${ }^{1,3}$, \\ Mohamed Abouhawwash ${ }^{3}$ \\ 1 Department of Mathematics, Sana'a University, Sana'a, Yemen \\ 2 Department of Mathematics, College of Sciences and Humanities in Aflaj, \\ Prince Sattam bin Abdulaziz University, Riyadh, Saudi Arabia \\ 3 Department of Mathematics, Faculty of Science, Mansoura University, Mansoura, Egypt
}

\begin{abstract}
In recent years, some generalized structures of topology were introduced. Supra topology was one the most important of those generalizations. To contribute in this orientation, we devoted this work to studying limit points and separation axioms on supra topological spaces by using supra $b$-open sets. We define them in a similar way of their counterparts on topological spaces. In general, we demonstrate their main properties and investigate the sufficient conditions for some equivalent relations between them. Some novel and interesting examples are provided.
\end{abstract}

2020 Mathematics Subject Classifications: 54A05, 54C08, 54D99

Key Words and Phrases: Supra $b$-open set, supra $b$ limit point, $S b T_{k}$-space $(k=0,1,2,3,4)$

\section{Introduction and preliminaries}

The term "Topology" on a nonempty set $\mathcal{U}$ is used to describe a subfamily of the power set $\mathcal{U}$ which is closed under arbitrary union and is closed under finite intersection. To model some real-life issues problems and keep some topological properties under conditions fewer than topology's conditions, various types of topology's extensions have been defined and discussed. One of the celebrated extensions of a topology is a supra topology defined by Mashhour et al. [24] in 1983. Then, Maki et al. [23] initiated the concept of minimal structures. Császár [17], in 2002, presented the concepts generalized topology and weak structure. Al-Odhari [1], in 2015, familiarized another extension of a topology called an infra topology.

In the pioneering work of Mashhour et al. [24] the concepts of supra continuity and supra separation axioms were studied. Following this work, many researchers have explored the topological concepts and notions in the frame of supra topology. For example, some classes of generalizations of open sets were furnished to supra topologies in

*Corresponding author.

DOI: https://doi.org/10.29020/nybg.ejpam.v15i1.4260

Email addresses: tareqalshami83@gmail.com (T.M. Al-shami), mhemdiabd@gmail.com (A. Mhemdi), mohjameel555@gmail.com (M. Jameel), saleh1284@mans.edu.eg (M. Abouhawwash) 
$[3,18,19,21,27,29]$. The class of supra $R$-open sets was studied in a topological spaces under the name of somewhere dense sets [4, 16]. Mustafa and Qoqazeh [26] applied supra $D$-sets to investigate new families of separation axioms. Mustafa [25] defined supra $b$ compact and supra $b$-Lindelof spaces. Then, Al-shami established the concepts of supra paracompactness [7], supra complete Hausdorffness and supra complete regularity [8]. In [9-11, 20], Al-shami with his coauthors scrutinized the main properties of limit points using the famous generalizations of supra open sets. Also, Al-shami with his coauthors $[2,5,6,12]$ introduced different kinds of supra compactness. The connected spaces were also initiated in supra topologies by the authors of $[22,28]$. As evidence of the importance of supra topologies, they were explored and discussed in the soft frames; see, [13-15].

It should be noted that some topology's properties are evaporated in supra topologies like the distribution property for the interior and closure operators with respect to the operations of intersection and union. Another example of these missing properties is the relationship between compact and closed subsets of Hausdorff space.

We organize the rest of this manuscript as follows. In Section (2), we recall the concepts and findings that make this work readable. In Section (3), we apply the class of supra $b$ open sets to display novel kinds of limit points of sets. We devoted Section (4) to introduce new families of spaces, namely $S b T_{k}$-spaces $(k=0,1,2,3,4)$. Finally, we provide some conclusions and suggest some future work in Section (5).

\section{Preliminaries}

Herein, we mention some concepts and findings given in the literature of supra topologies that are necessary to understand this article.

Definition 1. [24] We call a subfamily $\Omega$ of the power set of $\mathcal{U} \neq \emptyset$ a supra topology (in short, $S T)$ provided that $\mathcal{U} \in \Omega$ and the arbitrary union of members of $\Omega$ is also a member of $\Omega$.

We call a pair $(\mathcal{U}, \Omega)$ a supra topological space (in short, STS). The members of $\Omega$ are said to be supra open sets and the complement of each member of $\Omega$ is said to be a supra closed set.

Remark 1. (i) We call $\Omega$ an associated $S T$ with a topology $\tau$ if $\tau \subseteq \Omega$.

(ii) Henceforth, we consider $(\mathcal{U}, \Omega)$ and $(\mathcal{V}, \Psi)$ as associated STSs with the topological spaces $(\mathcal{U}, \tau)$ and $(\mathcal{V}, \theta)$, respectively.

Definition 2. [29] A subset $O$ of $(\mathcal{U}, \Omega)$ is said to be supra b-open if $O \subseteq \operatorname{int}(\operatorname{cl}(O)) \bigcup \operatorname{cl}(\operatorname{int}(O))$.

Definition 3. [18, 24] Let $O \subseteq \mathcal{U}$.

(i) The union of all supra open (respectively, supra b-open) subsets of an $S T S(\mathcal{U}, \Omega)$ contained in $O$ is denoted by int $^{\Omega}(O)$ (respectively, bint $\left.{ }^{\Omega}(O)\right)$. 
(ii) The intersection of all supra closed (respectively, supra b-closed) subsets of an STS $(\mathcal{U}, \Omega)$ including $O$ is denoted by $\mathrm{cl}^{\Omega}(O)$ (respectively, bcl ${ }^{\Omega}(O)$ ).

For simplicity, we sometimes write $\operatorname{int}(O), \operatorname{cl}(O), \operatorname{bint}(O)$ and $b c l(O)$ instead of $\operatorname{int}^{\Omega}(O)$, $c l^{\Omega}(O), \operatorname{bint}^{\Omega}(O)$ and $b c l^{\Omega}(O)$, respectively.

Definition 4. [18] Let $g:(\mathcal{U}, \Omega) \rightarrow(Y, \Psi)$ be a mapping.

(i) If the inverse image of every open set is supra b-open, then we call $g$ a supra bcontinuous mapping.

(ii) If the image of every open (respectively, closed) set is supra b-open (respectively, supra b-closed), then we call g a supra b-open (respectively, supra b-closed) mapping.

Definition 5. [2] Let $O$ be a subset of an $S T S(\mathcal{U}, \Omega)$. We call a class $\Omega_{A}=\{A \cap \Theta$ : $\Theta \in \Omega\}$ a supra relative topology on $O$, call $\left(O, \Omega_{A}\right)$ a supra subspace of $(\mathcal{U}, \Omega)$.

Definition 6. [12] We call $\beta$ a basis for an $S T S(\mathcal{U}, \Omega)$ if every element of $\Omega$ can be expressed as a union of elements of $\beta$.

Definition 7. [12] Let $\left\{\left(\mathcal{U}_{k}, \Omega_{k}\right): k=1,2, \ldots, n\right\}$ be a family of STSs. Then, $\left\{\Theta_{1} \times \Theta_{2} \times\right.$ $\left.\ldots \times \Theta_{n}: \Theta_{k} \in \Omega_{k}\right\}$ constitutes a basis for an $S T C$ on $\mathcal{U}=\prod_{k=1}^{n} \mathcal{U}_{k}$. We call $(\mathcal{U}, C)$ a finite product of STSs.

Proposition 1. [12] Let $O \subseteq(\mathcal{U}, \Omega)$ and $P \subseteq(\mathcal{V}, \Psi)$. Then, $\operatorname{int}(O \times P)=\operatorname{int}(O) \times \operatorname{int}(P)$ and $\operatorname{cl}(O \times P)=\operatorname{cl}(O) \times \operatorname{cl}(P)$.

\section{Supra $b$-limit points of a set}

We devote this section to defining a new type of supra limit points of a set by using supra $b$-open sets. With the help of examples, we probe their fundamental properties and describe their behaviors in some spaces.

Definition 8. We call $O$ a supra $b$ neighbourhood of $\xi$ in an $S T S(\mathcal{U}, \Omega)$ provided that there is a supra b-open set $F$ including $\xi$ such that $\xi \in F \subseteq O$.

Definition 9. We call $\xi \in \mathcal{U}$ a supra b-limit point of a subset $O$ of an $S T S(\mathcal{U}, \Omega)$ if every supra $b$ neighborhood $W$ of $\xi$ satisfying that $W \backslash\{\xi\} \bigcap A \neq \emptyset$.

A supra $b$ derived set of $O$, symbolized by $O^{b \prime}$, is all supra $b$-limit points of $O$.

One can prove the two results below easily, so we omit their proofs.

Proposition 2. For every $O, P \subseteq(\mathcal{U}, \Omega)$, we have $O \subseteq P$ implies $O^{b \prime} \subseteq P^{b \prime}$.

Corollary 1. Let $O$ and $P$ be subsets of $(\mathcal{U}, \Omega)$. Then,

(i) $O^{b \prime} \cup P^{b \prime} \subseteq(O \cup P)^{b \prime}$. 
(ii) $(O \cap P)^{b \prime} \subseteq O^{b \prime} \cap P^{b \prime}$.

The converse of the above proposition and corollary are in general false as the following example shows.

Example 1. Let $\Omega=\left\{\emptyset, \mathcal{U},\left\{\xi_{1}, \xi_{2}\right\},\left\{\xi_{2}, \xi_{3}\right\},\left\{\xi_{1}, \xi_{2}, \xi_{3}\right\}\right\}$ be a supra topology on $\mathcal{U}=$ $\left\{\xi_{1}, \xi_{2}, \xi_{3}, \xi_{4}\right\}$. Then $\left\{\emptyset, \mathcal{U},\left\{\xi_{2}\right\},\left\{\xi_{1}, \xi_{2}\right\},\left\{\xi_{2}, \xi_{3}\right\},\left\{\xi_{1}, \xi_{3}\right\},\left\{\xi_{2}, \xi_{4}\right\},\left\{\xi_{1}, \xi_{2}, \xi_{3}\right\},\left\{\xi_{1}, \xi_{2}, \xi_{4}\right\}\right.$, $\left.\left\{\xi_{1}, \xi_{3}, \xi_{4}\right\},\left\{\xi_{2}, \xi_{3}, \xi_{4}\right\}\right\}$ is the collection of all supra b-open subsets of $(\mathcal{U}, \Omega)$. If $O=$ $\left\{\xi_{1}, \xi_{4}\right\}, P=\left\{\xi_{2}, \xi_{3}\right\}, C=\left\{\xi_{1}, \xi_{2}, \xi_{4}\right\}$ and $D=\left\{\xi_{2}, \xi_{3}, \xi_{4}\right\}$, then $O^{b \prime}=\emptyset, P^{b /}=\left\{\xi_{1}\right\}$, $C^{b \prime}=\left\{\xi_{3}, \xi_{4}\right\}$ and $D^{b \prime}=\left\{\xi_{1}, \xi_{2}, \xi_{4}\right\}$. Now, we have the following cases:

(i) $O^{b \prime} \subseteq P^{b \prime}$, but $P \nsubseteq O$.

(ii) $O^{b \prime} \cup P^{b \prime}=\left\{\xi_{1}\right\}$ and $(O \bigcup P)^{b \prime}=\left\{\xi_{1}, \xi_{3}, \xi_{4}\right\}$. Then $(O \bigcup P)^{b \prime} \nsubseteq O^{b \prime} \cup P^{b \prime}$.

(iii) $C^{b \prime} \cap D^{b \prime}=\left\{\xi_{4}\right\}$ and $(C \bigcap D)^{b \prime}=\emptyset$. Then $C^{b \prime} \cap D^{b \prime} \nsubseteq(C \cap D)^{b \prime}$.

Proposition 3. Let $O$ be a subset of $(\mathcal{U}, \Omega)$ and $\xi \in \mathcal{U}$. Then $\xi \in O^{b \prime}$ iff $\xi \in(O \backslash\{\xi\})^{b \prime}$.

Proof. $\Rightarrow$ : Consider $\xi \in O^{b \prime}$. Then, $(\Theta \backslash\{\xi\}) \cap O \neq \emptyset$ for any supra $b$-open set $\Theta$ including $\xi$. Obviously, we obtain $(\Theta \backslash\{\xi\}) \bigcap(O \backslash\{\xi\}) \neq \emptyset$. This means that $\xi \in(O \backslash\{\xi\})^{b \prime}$. Proposition (2) proves the sufficient part.

Theorem 1. Let $O$ be a subset of $(\mathcal{U}, \Omega)$. Then.

(i) $O$ is a supra b-closed set iff $O^{b \prime} \subseteq O$.

(ii) $O \bigcup O^{b \prime}$ is a supra b-closed set.

(iii) $b c l(O)=O \bigcup O^{b \prime}$.

Proof.

(i) Let $O$ be a supra $b$-closed set and $\xi \notin O$. Since $O^{c} \bigcap O=\emptyset$ and $O^{c}$ is a supra $b$-open set including $\xi$, we obtain $\xi \notin O^{b \prime}$. Therefore, $O^{b \prime} \subseteq O$. Conversely, suppose that $\xi \in O^{c}$. Since $O^{b \prime} \subseteq O, \xi \notin O^{b \prime}$. Accordingly, we find a supra $b$-open set $\Theta_{\xi}$ satisfying $\Theta_{\xi} \backslash\{\xi\} \cap O=\emptyset$. Now, $\Theta_{\xi} \cap O=\emptyset$ because $\xi \in O^{c}$. Therefore, $\Theta_{\xi} \subseteq O^{c}$, which means that $O^{c}=\bigcup\left\{\Theta_{\xi}: \xi \in O^{c}\right\}$. This ends the proof that $O$ is supra $b$-closed.

(ii) Suppose that $\xi \notin\left(O \bigcup O^{b \prime}\right)$. Then, $\xi \notin O$ and $\xi \notin O^{b \prime}$. This means that there exists a supra $b$-open set $\Theta$ satisfying the following equality.

$$
\Theta \bigcap O=\emptyset
$$

Now, for every $\xi \in \Theta$, we obtain $\xi \notin O^{b \prime}$. So we obtain the following equality.

$$
\Theta \bigcap O^{b \prime}=\emptyset
$$

It comes from (1) and (2) that $\Theta \bigcap\left(O \bigcup O^{b \prime}\right)=\emptyset$. Thus, $\xi \notin\left(O \bigcup O^{b \prime}\right)^{b \prime}$. Hence, $\left(O \bigcup O^{b \prime}\right)^{b \prime} \subseteq\left(O \bigcup O^{b \prime}\right)$. According to (i), $O \bigcup O^{b \prime}$ is supra $b$-closed. 
(iii) It is clear that $O \bigcup O^{b \prime} \subseteq \operatorname{bcl}(O)$. Conversely, it well known that $b c l(O)$ is the smallest supra $b$-closed set including $O$. Since $O \bigcup O^{b \prime}$ is a supra $b$-closed set including $O$, $b c l(O) \subseteq O \bigcup O^{b \prime}$. Hence, $b c l(O)=O \bigcup O^{b \prime}$.

Corollary 2. If $O$ is a supra b-closed subset of $(\mathcal{U}, \Omega)$, then $O^{b \prime},\left(O^{b \prime}\right)^{b \prime},\left(\left(O^{b \prime}\right)^{b \prime}\right)^{b \prime}, \ldots$ are supra b-closed sets.

Definition 10. We call a mapping $g:(\mathcal{U}, \Omega) \rightarrow(\mathcal{V}, \Psi)$ :

(i) supra $b^{\star}$-continuous if $g^{-1}(H)$ is supra b-open for any supra b-open set $H$.

(ii) supra $b^{\star}$-open (respectively, supra $b^{\star}$-closed) if $g(H)$ is supra b-open (respectively, supra b-closed) for any supra b-open (respectively, supra b-closed) set $H$.

We call a bijective, supra $b^{\star}$-continuous and supra $b^{\star}$-open mapping a supra $b^{\star}$-homeomorphism.

Theorem 2. If $f:(\mathcal{U}, \Omega) \rightarrow(\mathcal{V}, \Psi)$ is a supra $b^{\star}$-homeomorphism mapping, then $f\left(O^{b \prime}\right)=$ $(g(O))^{b \prime}$ for each $O \subseteq \mathcal{U}$.

Proof. Consider $\xi \notin(f(O))^{b \prime}$. Then, $(H \backslash\{\xi\}) \cap f(O)=\emptyset$ for some supra $b$-open sets $H$ including $\xi$. Directly, $f^{-1}[(H \backslash\{\xi\}) \bigcap f(O)]=f^{-1}(\emptyset)$. Now, $\left(f^{-1}(H) \backslash f^{-1}(\xi)\right) \bigcap O=\emptyset$. This means that $f^{-1}(\xi) \notin O^{b \prime}$. The bijectiveness of $f$ implies that $\xi \notin f\left(O^{b \prime}\right)$. Thus, $f\left(O^{b \prime}\right) \subseteq(f(O))^{b \prime}$. Following similar technique, we obtain $(f(O))^{b \prime} \subseteq f\left(O^{b \prime}\right)$.

Definition 11. A sub-collection $\Lambda$ of the power set of $\mathcal{U} \neq \emptyset$ is said to have the difference property if $\Theta \in \Lambda$ implies that $\Theta \backslash\{\xi\} \in \Lambda$.

To illustrate the difference property, we supply the next two examples.

Example 2. Consider $\Omega=\{\emptyset, \Theta \subseteq \mathcal{N}: \Theta$ is infinite $\}$ as an $S T$ on the natural numbers set $\mathcal{N}$. Obviously, $\Theta \in \Omega$ implies $\Theta \backslash\{\xi\} \in \Omega$. Then, $(\mathcal{N}, \Omega)$ has the difference property. Note that the families of supra open and supra b-open subsets of $(\mathcal{N}, \Omega)$ are identical. Hence, $(\mathcal{N}, \Omega)$ has the difference property for the family of supra b-open sets.

Example 3. Consider $\Omega=\{\emptyset, \mathcal{N} \backslash\{1,3\}, \mathcal{N} \backslash\{1,4\}, \mathcal{N} \backslash\{2,3\}, \mathcal{N} \backslash\{2,4\}\} \bigcup\{\Theta \subseteq \mathcal{N}: \Theta$ such that $\{1,2\} \subseteq \Theta$ or $\{3,4\} \subseteq \Theta\}$ as an $S T$ on the natural numbers set $\mathcal{N}$. Then $\{1,2\} \in \Omega$, but $\{1,2\} \backslash\{2\}=\{1\} \notin \Omega$. Thus, $(\mathcal{U}, \Omega)$ does not have the difference property.. Hence, it does not have the difference property for the family of supra b-open sets.

Theorem 3. Let $O$ be a subset of $(\mathcal{U}, \Omega)$ which has the difference property for the family of supra b-open sets. Then.

(i) $\left(O^{b \prime}\right)^{b \prime} \subseteq O^{b \prime}$.

(ii) $b c l\left(O^{b \prime}\right)=O^{b \prime}=(b c l(O))^{b \prime}$.

(iii) $O^{b \prime}=\emptyset$ if $O$ is finite. 
Proof.

(i) Consider $\xi \notin O^{b \prime}$. Then, $\Theta \backslash\{\xi\} \bigcap O=\emptyset$ for some supra $b$-open sets $\Theta$ including $\xi$. By the difference property for the family of supra $b$-open subsets of $(\mathcal{U}, \Omega)$, we obtain $\Theta \backslash\{\xi\}$ is a supra $b$-open set. So that, $\Theta \backslash\{\xi\} \bigcap O^{b \prime}=\emptyset$. Now, $\Theta \bigcap\left(O^{b \prime}\right)^{b \prime}=\emptyset$ because $\xi \notin O^{b \prime}$. Therefore, $\xi \notin\left(O^{b \prime}\right)^{b \prime}$. Hence, $\left(O^{b \prime}\right)^{b \prime} \subseteq O^{b \prime}$.

(ii) Since $\left(O^{b \prime}\right)^{b \prime} \subseteq O^{b \prime}$, it comes from Theorem (1) that $O^{b \prime}$ is supra b-closed. Thus,

$$
\operatorname{bcl}\left(O^{b \prime}\right)=O^{b \prime}
$$

Also, $(O)^{b \prime} \subseteq(b c l(O))^{b \prime}$ because $O \subseteq b c l(O)$. Conversely, let $\xi \notin(O)^{b \prime}$. Then, it comes from 1 above that $\Theta \backslash\{\xi\} \cap O=\emptyset$ and $\Theta \backslash\{\xi\} \cap O^{b \prime}=\emptyset$. This means that $\Theta \backslash\{\xi\} \bigcap b c l(O)=\emptyset$. Therefore, $\xi \notin(b c l(O))^{b \prime}$. Thus, $(b c l(O))^{b \prime} \subseteq(O)^{b \prime}$. Hence

$$
(b c l(O))^{b \prime}=(O)^{b \prime}
$$

Equalities (3) and (4) end the proof.

(iii) Consider $O$ is a finite subset of $\mathcal{U}$. Suppose that $\xi \in \mathcal{U}$ such that $\xi \in O^{b \prime}$. Then, $\Theta \backslash\{\xi\} \cap O \neq \emptyset$ for every supra $b$-open set $\Theta$ including $\xi$. Now, for every $\zeta \in O$ such that $\zeta \neq \xi$, we have $\Theta \backslash\{\xi, \zeta\}$ is a supra $b$-open set. Thus, $\Theta \backslash[O \bigcup\{\xi\}]$ is a supra $b$-open set such that $\Theta \backslash[O \bigcup\{\xi\}] \cap O=\emptyset$. This implies that $\xi \notin O^{b \prime}$. But this is a contradiction. Hence, $O^{b \prime}=\emptyset$.

To show that the converse of Theorem 3 is not true if $(\mathcal{U}, \Omega)$ does not have the difference property for the family of supra $b$-open sets, consider $O=\{2,4,5\}$ as a subset of STS furnished in Example (3). Note that the families of supra open and supra $b$-open sets are identical. So that, we obtain $O^{b \prime}=\mathcal{N} \backslash\{2,4\},\left(O^{b \prime}\right)^{b \prime}=\mathcal{N} \backslash\{1,3\}$ and $\operatorname{cl}\left(O^{b \prime}\right)=\mathcal{N}$, which means that

(i) $\left(O^{b \prime}\right)^{b \prime} \nsubseteq O^{b \prime}$.

(ii) $b c l\left(O^{b \prime}\right) \neq O^{b \prime}$.

(iii) $O^{b \prime} \neq \emptyset$ in spite of $O$ is finite.

\section{Supra $b$ separation axioms}

In this section, we familiarize the concepts of regularity, normality and $T_{k}$-spaces using supra $b$-open sets. We give them some descriptions and reveal the interrelations between them with the assistant of examples and counterexamples.

Definition 12. An $S T S(\mathcal{U}, \Omega)$ is said to be:

(i) $S b T_{0}$ if for every $\xi \neq \zeta \in \mathcal{U}$, there is a supra b-open set $O$ such that $\xi \in O$ or $\zeta \in O$. 
A. Mhemdi et al. / Eur. J. Pure Appl. Math, 15 (1) (2022), 15-29

(ii) $S b T_{1}$ if for every $\xi \neq \zeta \in \mathcal{U}$, there are supra b-open sets $O, \Theta$ such that $\xi \in O \backslash \Theta$ and $\zeta \in \Theta \backslash O$.

(iii) $S b T_{2}$ (or, supra $b$ Hausdorff) if for every $\xi \neq \zeta \in \mathcal{U}$, there are disjoint supra b-open sets $O, \Theta$ such that $\xi \in O$ and $\zeta \in \Theta$.

(iv) supra $b$ regular if for every supra $b$-closed set $F$ and each $\xi \notin F$, there are disjoint supra b-open sets $O, \Theta$ such that $\xi \in O$ and $F \subseteq \Theta$.

(v) supra $b$ normal if for every two disjoint supra b-closed sets are separated by two disjoint supra b-open sets.

(vi) $\mathrm{SbT}_{3}$ (respectively, $S b T_{4}$ ) if it is supra b regular (respectively, supra b normal) and $S b T_{1}$.

Theorem 4. The next three properties are identical.

(i) $(\mathcal{U}, \Omega)$ is $S b T_{0}$;

(ii) $\operatorname{bcl}(\{\xi\}) \neq \operatorname{bcl}(\{\zeta\})$ for every $\xi \neq \zeta \in \mathcal{U}$;

(iii) $\{\xi\}^{b \prime}$ is a union of supra b-closed sets for every $\xi \in \mathcal{U}$.

Proof. $1 \rightarrow 2$ : Let $\xi \neq \zeta \in \mathcal{U}$. Then there is a supra $b$-open set $O$ such that $\xi \in \Omega$ or $\zeta \in \Omega$. Say, $\xi \in \Theta$ and $\zeta \notin \Theta$. Since $\Theta$ is a supra $b$-open set including $\xi$ such that $\Theta \bigcap\{\zeta\}=\emptyset$, we obtain $\xi \notin b c l(\{\zeta\})$. Obviously, $\xi \in \operatorname{bcl}(\{\xi\})$; hence, $\operatorname{bcl}(\{\xi\}) \neq b c l(\{\zeta\})$.

$2 \rightarrow 3$ : Suppose that $\zeta \in\{\xi\}^{b \prime}$. Then, $\zeta \in b c l(\{\xi\})$. Consequently, bcl $(\zeta) \subseteq b c l(\{\xi\})$. This means that $\zeta \in b c l(\zeta) \subseteq\{\xi\}^{b \prime}$. So that, $\{\xi\}^{b \prime}=\bigcup\left\{b c l(\zeta)\right.$ : for every $\left.\zeta \in\{\xi\}^{b \prime}\right\}$.

$3 \rightarrow 1$ : Consider $\xi \neq \zeta$. Then

(i) Either $\zeta \in\{\xi\}^{b \prime}$. Then there is a supra $b$-closed set $F$ such that $\zeta \in F \subseteq\{\xi\}^{b \prime}$. This means that $\xi \notin F$. Thus, $F^{c}$ is a supra $b$-open set including $\xi$ such that $\zeta \notin F^{c}$.

(ii) $\operatorname{Or} \zeta \notin\{\xi\}^{b \prime}$. Then there is a supra $b$-open set $\Theta$ including $\zeta$ such that $\xi \notin \Theta$.

It follows from the above two cases that $(\mathcal{U}, \Omega)$ is $S b T_{0}$.

Corollary 3. In an $S b T_{0}$-space $(\mathcal{U}, \Omega)$, there exists at most a singleton set which is a supra $b$ dense $(\{\xi\}$ is a supra $b$ dense set if $b c l\{\xi\}=\mathcal{U})$.

Proof. Suppose that $\{\xi\}$ and $\{\zeta\}$ are two distinct singleton subset of an $S b T_{0}$-space $(\mathcal{U}, \Omega)$ such that $\operatorname{bcl}(\{\xi\})=\operatorname{bcl}(\{\zeta\})=\mathcal{U}$. This leads to a contradiction because $(\mathcal{U}, \Omega)$ is $S b T_{0}$. Hence, we obtain the desired result.

Theorem 5. The next there properties are identical.

(i) $(\mathcal{U}, \Omega)$ is $S b T_{1}$; 
(ii) All singleton subsets of $(\mathcal{U}, \Omega)$ are supra $b$-closed;

(iii) The intersection of all supra b-open sets including a set $O$ is exactly $O$;

(iv) $\{\xi\}^{b \prime}=\emptyset$ for every $\xi \in \mathcal{U}$.

Proof. $1 \rightarrow 2$ : Let $(\mathcal{U}, \Omega)$ be $S b T_{1}$ such that $\{\xi\} \subseteq \mathcal{U}$. For each $\zeta \neq \xi \in \mathcal{U}$, there is a supra $b$-open set $\Theta$ including $\zeta$ satisfies that $\Theta \bigcap\{\xi\}=\emptyset$. Then, $\zeta \notin b c l(\{\xi\})$. Thus, $\operatorname{bcl}(\{\xi\})=\{\xi\}$. Hence, we obtain the required result.

$2 \rightarrow 3$ : Let $O \subseteq \mathcal{U}$ and $\xi \in O^{c}$. Then $\{\xi\}^{c}$ is a supra $b$-open set including $O$. Therefore, $O \subseteq\{\Theta: \Theta$ is a supra $b$-open set including $O\} \subseteq\left\{\{\xi\}^{c}: \xi \in O^{c}\right\} \subseteq O$. Hence, $O=\{\Theta: \Theta$ is a supra $b$-open set including $O\}$.

$3 \rightarrow 4$ : Let $\xi \in \mathcal{U}$ such that $\{\xi\}^{b \prime} \neq \emptyset$. Then there is $\zeta \neq \xi$ such that $\zeta \in\{\xi\}^{b \prime}$. So that, $\Theta \backslash\{\zeta\} \bigcap\{\xi\} \neq \emptyset$ for every supra $b$-open set $\Theta$ including $\zeta$. This contradicts 3 because the intersection of all supra $b$-open sets including $\zeta$ is not equal $\{\zeta\}$. Hence, $\{\xi\}^{b \prime}=\emptyset$.

$4 \rightarrow 1$ : Let $\xi \neq \zeta$. Then, the singleton sets $\{\xi\}$ and $\{\zeta\}$ are supra $b$-closed because $\{\xi\}^{b \prime}=\emptyset$ and $\{\zeta\}^{b \prime}=\emptyset$. This implies that $\{\xi\}^{c}$ and $\{\zeta\}^{c}$ are supra $b$-open sets including $\{\zeta\}$ and $\{\xi\}$, respectively. Hence, $(\mathcal{U}, \Omega)$ is $S b T_{1}$.

Proposition 4. If $(\mathcal{U}, \Omega)$ satisfies the difference property for the family of supra b-open sets, then it is $S b T_{1}$.

Proof. Since $\mathcal{U}$ is a supra $b$-open set and $(\mathcal{U}, \Omega)$ satisfies the difference property for the family of supra $b$-open sets, we find that $\mathcal{U} \backslash\{\xi\}$ is a supra $b$-open set for each $\xi \in \mathcal{U}$. Hence, we obtain the desired result.

To clarify that the converse of the aforementioned proposition is in general false, we furnish the example below.

Example 4. Every singleton subset of $(\mathcal{U}, \Omega)$, given in Example (1), is supra b-closed; therefore, $(\mathcal{U}, \Omega)$ is $S b T_{1}$. In contrast, a set $\left\{\xi_{1}, \xi_{3}\right\}$ is supra b-open, whereas $\left\{\xi_{1}, \xi_{3}\right\} \backslash\left\{\xi_{1}\right\}$ is not supra b-open. Consequently, $(\mathcal{U}, \Omega)$ does not have the difference property for the class of supra b-open sets.

The next definition will help to prove that $S b T_{0^{-}}$and $S b T_{1}$-spaces are equivalent.

Definition 13. We call $(\mathcal{U}, \Omega)$ a supra b symmetric space if $\xi \in$ bcl $\{\zeta\}$ implies that $\zeta \in b c l\{\xi\}$ for $\xi \neq \zeta \in \mathcal{U}$.

Theorem 6. Let $(\mathcal{U}, \Omega)$ be a supra b symmetric space. Then it is $S b T_{1}$ iff it is $S b T_{0}$.

Proof. $\Rightarrow$ : It is straightforward.

$\Leftarrow$ : For two distinct points $\xi$ and $\zeta$, there is a supra $b$-open set $\Theta$ including only one of them. Assume that $\xi \in \Theta$ and $\zeta \notin \Theta$. Then, $\xi \notin b c l\{\zeta\}$. Now, $\zeta \notin b c l\{\xi\}$ because $(\mathcal{U}, \Omega)$ is supra $b$ symmetric. Thus, $(b c l\{\xi\})^{c}$ is a supra $b$-open set including $b$. Hence, $(\mathcal{U}, \Omega)$ is $S b T_{1}$. 
Theorem 7. The next three properties are identical.

(i) $(\mathcal{U}, \Omega)$ is $S b T_{2}$;

(ii) $\{\xi\}=\bigcap\left\{F_{k}: F_{k}\right.$ is a supra b-closed neighborhood of $\left.\xi\right\}$ for every $\xi \in \mathcal{U}$;

(iii) $\triangle=\{(\xi, \xi): \xi \in \mathcal{U}\}$ forms a supra $b$-closed set in the product of supra spaces $\mathcal{U} \times \mathcal{U}$.

Proof. $1 \rightarrow 2$ : Since $(\mathcal{U}, \Omega)$ is $S b T_{2}$, for $\xi \neq \zeta$ there are disjoint supra $b$-open sets $\Theta_{k}$ and $O_{k}$ such that $\xi \in \Theta_{k}$ and $\zeta \in O_{k}$. Then, $\xi \in b c l\left(\Theta_{k}\right) \subseteq O_{k}^{c}=F_{k}$. Therefore, $F_{k}$ is a supra $b$-closed neighborhood of $\xi$ such that $\zeta \notin F_{k}$. Thus, $\{\xi\}=\bigcap\left\{F_{k}: F_{k}\right.$ is a supra $b$-closed neighborhood of $\xi\}$.

$2 \rightarrow 1$ : Consider $\xi \neq \zeta$. Since $\{\xi\}=\bigcap\left\{F_{k}: F_{k}\right.$ is a supra $b$-closed neighborhood of $\xi\}$, there is a supra $b$-closed neighborhood $F_{k_{0}}$ of $\xi$ such that $\zeta \notin F_{k_{0}}$. So that, there is a supra $b$-open set $\Theta$ including $\xi$ such that $\xi \in b c l(\Theta) \subseteq F_{k_{0}}$. Obviously, $(b c l(\Theta))^{c}$ is a supra $b$-open set including $\zeta$ and $\Theta \bigcap(b c l(\Theta))^{c}=\emptyset$. Hence, $(\mathcal{U}, \Omega)$ is $S b T_{2}$.

$1 \rightarrow 3$ : Suppose that $(\xi, \zeta) \in \mathcal{U} \times \mathcal{U}-\triangle$. Then $\xi \neq \zeta$. By hypothesis, there are disjoint supra $b$-open sets $\Theta$ and $O$ respectively including $\xi$ and $\zeta$. Now, $(\xi, \zeta) \in \Theta \times O \subseteq \mathcal{U} \times \mathcal{U}-\triangle$, which proves that $\mathcal{U} \times \mathcal{U}-\triangle$ is a supra $b$ neighbourhood of any of its points. Thus, $\triangle$ is supra $b$-closed.

$3 \rightarrow 1$ : Consider $\triangle$ as a supra $b$-closed subset of $\mathcal{U} \times \mathcal{U}$. For $\xi \neq \zeta \in \mathcal{U}$, we have $\mathcal{U} \times \mathcal{U}-\triangle$ is a supra $b$-open set including $(\xi, \zeta)$. So that, there are supra $b$-open subsets $\Theta$ and $O$ of $(\mathcal{U}, \Omega)$ such that $(\xi, \zeta) \in \Theta \times O \subseteq \mathcal{U} \times \mathcal{U}-\triangle$. Thus, $\Theta$ and $O$ are disjoint supra $b$-open sets respectively including $\xi$ and $\zeta$. This ends the proof that $(\mathcal{U}, \Omega)$ is $S b T_{2}$.

Theorem 8. The next three properties are identical.

(i) $(\mathcal{U}, \Omega)$ is a supra $b$ regular space;

(ii) For every supra b-open subset $O$ of $(\mathcal{U}, \Omega)$ including $\xi$, there is a supra b-open subset $V$ of $(\mathcal{U}, \Omega)$ such that $\xi \in V \subseteq b c l(V) \subseteq O$;

(iii) Every supra b-open subset $O$ of $(\mathcal{U}, \Omega)$ is written: $O=\bigcup\{H: H$ is a supra b-open subset of $(\mathcal{U}, \Omega)$ and $b c l(H) \subseteq O\}$.

Proof. $1 \rightarrow 2$ : Let $O$ be a supra $b$-open set such that $\xi \in U$. By hypothesis, there it disjoint supra $b$-open sets $V$ and $W$ respectively including $\xi$ and $O^{c}$. So that, $\xi \in V \subseteq$ $W^{c} \subseteq O$. Hence, $\xi \in V \subseteq b c l(V) \subseteq O$.

$2 \rightarrow 3$ : Let $O$ be a supra $b$-open set. By hypothesise, for each $\xi \in O$, there exists a supra $b$-open set $H$ such that $\xi \in H \subseteq b c l(H) \subseteq O$. Thus, $O=\bigcup\{H: H$ is supra $b$-open and $b c l(H) \subseteq O\}$.

$3 \rightarrow 1$ : Suppose that $F$ is a supra $b$-closed set such that $\xi \notin F$. Then $F^{c}=\bigcup\{H: H$ is supra $b$-open and $\left.b c l(H) \subseteq F^{c}\right\}$. Since $\xi \in F^{c}$, there is a supra $b$-open set $H_{\xi}$ including $\xi$ such that $b c l\left(H_{\xi}\right) \subseteq F^{c}$. Put $V=\left(b c l\left(H_{\xi}\right)\right)^{c}$; this means that $V$ is a supra $b$-open set including $F$. Thus, $V \cap H_{\xi}=\emptyset$, which finishes the proof. 
Theorem 9. The concepts of $S b T_{2}, S b T_{1}$ and $S b T_{0}$ are equivalent under a supra $b$ regular space.

Proof. The directions $S b T_{2} \Rightarrow S b T_{1} \Rightarrow S b T_{0}$ are clear.

To obtain the desired result, we prove that $S b T_{0} \Rightarrow S b T_{2}$. To this end, let $\xi \neq \zeta \in \mathcal{U}$. It follows from Theorem (4) that $b c l\{\xi\} \neq b c l\{\zeta\}$. So that, $\xi \notin b c l\{\zeta\}$ or $\zeta \notin b c l\{\xi\}$. Consider $\xi \notin b c l\{\zeta\}$. By the condition of supra $b$ regularity, there are disjoint supra $b$-open sets $\Theta$ and $O$ respectively including $\xi$ and $b c l\{\zeta\}$. Hence, the proof is complete.

Theorem 10. The next properties are identical.

(i) $(\mathcal{U}, \Omega)$ is a supra b normal space;

(ii) For every supra $b$-closed set $F$ and supra b-open set $O$ including $F$, there is a supra b-open set $V$ such that $F \subseteq V \subseteq b c l(V) \subseteq O$;

(iii) For every supra b-open sets $O$ and $V$ such that $O \bigcup V=\mathcal{U}$, there are two supra $b$-closed sets $F$ and $H$ respectively included in $O$ and $V$ such that $F \cup H=\mathcal{U}$.

Proof. $1 \rightarrow 2$ : Let $(\mathcal{U}, \Omega)$ be supra $b$ normal and $F$ be a supra $b$-closed subset of a supra $b$-open set $O$. Then $O^{c}$ and $F$ are disjoint supra $b$-closed sets. So that, there are two disjoint supra $b$-open sets $W$ and $V$ respectively including $O^{c}$ and $F$. Now, $F \subseteq V \subseteq W^{c}=b c l\left(W^{c}\right) \subseteq O$, which means that $F \subseteq V \subseteq b c l(V) \subseteq O$.

$2 \rightarrow 3$ : Let $O$ and $V$ be supra $b$-open sets such that $O \bigcup V=\mathcal{U}$. Then, $O^{c}$ is a supra $b$-closed sets such that $O^{c} \subseteq V$. By 2, there is a supra $b$-open set $\Theta$ such that $O^{c} \subseteq \Theta \subseteq b c l(\Theta) \subseteq V$. Hence, $\Theta^{c} \subseteq O$ and $b c l(\Theta) \subseteq V$ are supra $b$-closed sets such that $\Theta^{c} \bigcup b c l(\Theta)=\mathcal{U}$.

$3 \rightarrow 1$ : Let $F$ and $H$ be disjoint supra $b$-closed sets. Since $F^{c}$ and $H^{c}$ are supra open sets such that $F^{c} \cup H^{c}=\mathcal{U}$, then there are two supra $b$-closed sets $M$ and $N$ such that $M \subseteq F^{c}, N \subseteq H^{c}$ and $M \bigcup N=\mathcal{U}$. Thus, $M^{c}$ and $N^{c}$ are two disjoint supra $b$-open sets respectively including $F$ and $H$. This ends the proof that $(\mathcal{U}, \Omega)$ is supra $b$ normal.

Theorem 11. Every $S b T_{k}$-space is $S b T_{k-1}$ for $k=1,2,3,4$.

The four examples below elucidate that the converse of Theorem (11) is in general false.

Example 5. Let $\Omega=\left\{\emptyset, \mathcal{U},\left\{\xi_{4}\right\},\left\{\xi_{1}, \xi_{3}, \xi_{4}\right\},\left\{\xi_{2}, \xi_{3}, \xi_{4}\right\},\left\{\xi_{1}, \xi_{2}, \xi_{4}\right\}\right\}$ be an $S T$ on $\mathcal{U}=$ $\left\{\xi_{1}, \xi_{2}, \xi_{3}, \xi_{4}\right\}$. Then the class of all supra b-open subsets of $(\mathcal{U}, \Omega)$ is $\left\{\emptyset, \mathcal{U},\left\{\xi_{4}\right\},\left\{\xi_{1}, \xi_{4}\right\}\right.$, $\left.\left\{\xi_{2}, \xi_{4}\right\},\left\{\xi_{3}, \xi_{4}\right\},\left\{\xi_{1}, \xi_{3}, \xi_{4}\right\},\left\{\xi_{2}, \xi_{3}, \xi_{4}\right\},\left\{\xi_{1}, \xi_{2}, \xi_{4}\right\}\right\}$. Therefore, $(\mathcal{U}, \Omega)$ is not an $S b T_{1}$ space because $\xi_{1} \neq \xi_{4}$ and all supra b-open sets including $\xi_{1}$ contain $\xi_{4}$ as well. On the other hand, it can be checked that $(\mathcal{U}, \Omega)$ is $S b T_{0}$.

Example 6. Since all singleton subsets of $(\mathcal{U}, \Omega)$, given in Example $(1)$, are supra bclosed, $(\mathcal{U}, \Omega)$ is $S b T_{1}$. In contrast, $(\mathcal{U}, \Omega)$ is not $S b T_{2}$ because $\xi_{1} \neq \xi_{3}$ and there do not exist disjoint supra b-open sets such that one of them contains $\xi_{1}$ and the other contains $\xi_{3}$. 
Example 7. Let $\Omega=\left\{\emptyset, \mathcal{U},\left\{\xi_{1}, \xi_{2}\right\},\left\{\xi_{3}, \xi_{4}\right\},\left\{\xi_{1}, \xi_{3}\right\},\left\{\xi_{2}, \xi_{4}\right\},\left\{\xi_{2}, \xi_{3}\right\},\left\{\xi_{1}, \xi_{2}, \xi_{3}\right\},\left\{\xi_{1}, \xi_{2}, \xi_{4}\right\}\right.$, $\left.\left\{\xi_{1}, \xi_{3}, \xi_{4}\right\},\left\{\xi_{2}, \xi_{3}, \xi_{4}\right\}\right\}$ be an $S T$ on $\mathcal{U}=\left\{\xi_{1}, \xi_{2}, \xi_{3}, \xi_{4}\right\}$. In $(\mathcal{U}, \Omega)$, a set is supra open iff it is supra b-open. Now, $\left\{\xi_{1}, \xi_{4}\right\}$ is a supra b-closed set and $\xi_{2} \notin\left\{\xi_{1}, \xi_{4}\right\}$. Since there do not exist two disjoint supra b-open sets such that one of them contains $\xi_{2}$ and the other contains $\left\{\xi_{1}, \xi_{4}\right\}$, we obtain $(\mathcal{U}, \Omega)$ is not $S b T_{3}$. In contrast, one can check that $(\mathcal{U}, \Omega)$ is $\mathrm{SbT}_{2}$.

Example 8. Let $\Omega=\left\{\emptyset, \mathcal{U},\left\{\xi_{2}\right\},\left\{\xi_{4}\right\},\left\{\xi_{2}, \xi_{4}\right\},\left\{\xi_{1}, \xi_{3}\right\},\left\{\xi_{1}, \xi_{4}\right\},\left\{\xi_{1}, \xi_{5}\right\},\left\{\xi_{2}, \xi_{3}\right\},\left\{\xi_{2}, \xi_{5}\right\}\right.$, $\left\{\xi_{3}, \xi_{5}\right\},\left\{\xi_{4}, \xi_{5}\right\},\left\{\xi_{1}, \xi_{2}, \xi_{3}\right\},\left\{\xi_{1}, \xi_{2}, \xi_{4}\right\},\left\{\xi_{1}, \xi_{2}, \xi_{5}\right\},\left\{\xi_{1}, \xi_{3}, \xi_{4}\right\},\left\{\xi_{1}, \xi_{3}, \xi_{5}\right\},\left\{\xi_{1}, \xi_{4}, \xi_{5}\right\},\left\{\xi_{2}\right.$, $\left.\xi_{3}, \xi_{4}\right\},\left\{\xi_{2}, \xi_{3}, \xi_{5}\right\},\left\{\xi_{2}, \xi_{4}, \xi_{5}\right\},\left\{\xi_{3}, \xi_{4}, \xi_{5}\right\},\left\{\xi_{1}, \xi_{2}, \xi_{3}, \xi_{4}\right\},\left\{\xi_{1}, \xi_{2}, \xi_{3}, \xi_{5}\right\},\left\{\xi_{1}, \xi_{2}, \xi_{4}, \xi_{5}\right\},\left\{\xi_{1}\right.$, $\left.\left.\xi_{3}, \xi_{4}, \xi_{5}\right\},\left\{\xi_{2}, \xi_{3}, \xi_{4}, \xi_{5}\right\}\right\}$ be an $S T$ on $\mathcal{U}=\left\{\xi_{1}, \xi_{2}, \xi_{3}, \xi_{4}, \xi_{5}\right\}$. In $(\mathcal{U}, \Omega)$, a set is supra open iff it is supra b-open. Now, $\left\{\xi_{1}, \xi_{2}\right\}$ and $\left\{\xi_{3}, \xi_{4}\right\}$ are disjoint supra b-closed subsets of $(\mathcal{U}, \Omega)$. Since there do not exist two disjoint supra b-open sets such that one of them contains $\left\{\xi_{1}, \xi_{2}\right\}$ and the other contains $\left\{\xi_{3}, \xi_{4}\right\}$, we obtain $(\mathcal{U}, \Omega)$ is not supra $b$ normal. Hence, it is not $S b T_{4}$. In contrast, one can check that $(\mathcal{U}, \Omega)$ is $S b T_{3}$.

Theorem 12. Every $S T_{k}$-space $(\mathcal{U}, \Omega)$ is $S b T_{k}$ for $k=0,1,2$.

Proof. Straightforward.

The converse of the above theorem is in general false as the next example shows.

Example 9. Let $\Omega=\left\{\emptyset, \mathcal{U},\left\{\xi_{1}\right\},\left\{\xi_{2}, \xi_{3}\right\}\right\}$ be an $S T$ on $\mathcal{U}=\left\{\xi_{1}, \xi_{2}, \xi_{3}\right\}$. One can check that $(\mathcal{U}, \Omega)$ is $S b T_{4}$ but is not $S T_{4}$.

Definition 14. Let $O \neq \emptyset$ be a subset of $(\mathcal{U}, \Omega)$. We call a class $\Omega_{O}=\{O \bigcap \Theta: \Theta$ is a supra b-open subset of $(\mathcal{U}, \Omega)\}$ a relative b-topology on $O$, and call $\left(O, \Omega_{A}\right)$ a b-subspace of $(\mathcal{U}, \Omega)$.

Definition 15. We call a property a relative b-hereditary if the it passes from an STS to every relative b-subspace.

One can prove the next two propositions easily; so we omit their proofs.

Proposition 5. Let $\left(Y, \Omega_{Y}\right)$ be an b-subspace of $(\mathcal{U}, \Omega)$. A subset $H$ of $Y$ is supra b-closed in $\left(Y, \Omega_{Y}\right)$ iff there exists a supra b-closed subset $F$ of $(\mathcal{U}, \Omega)$ such that $H=Y \cap F$.

Proposition 6. A property of being an $S b T_{k}$-space is a relative b-hereditary for $k=$ $0,1,2,3$.

Proposition 7. Let $g:(\mathcal{U}, \Omega) \rightarrow(\mathcal{V}, \theta)$ be an injective supra b-continuous mapping. If $(\mathcal{V}, \theta)$ is $T_{k}$, then $(\mathcal{U}, \Omega)$ is $S b T_{k}$ for $k=0,1,2$.

Proof. We give a proof when $k=2$.

Let $\xi \neq \zeta \in \mathcal{U}$. Since $g$ is injective, there are $x \neq y \in \mathcal{V}$ such that $x=f(\xi)$ and $y=f(\zeta)$. Since $(\mathcal{V}, \theta)$ is $T_{2}$, there are two disjoint open subsets $M$ and $N$ of $(\mathcal{V}, \theta)$ respectively including $x$ and $y$. Thus, $g^{-1}(M)$ and $g^{-1}(N)$ are disjoint supra $b$-open subsets of $(\mathcal{U}, \Omega)$ respectively including $\xi$ and $\zeta$. This proves that $(\mathcal{U}, \Omega)$ is $S b T_{2}$.

Similarly, one can prove the next findings. 
Proposition 8. Let $g:(\mathcal{U}, \tau) \rightarrow(\mathcal{V}, \Psi)$ be a bijective supra b-open mapping. If $(\mathcal{U}, \tau)$ is $T_{k}$, then $(\mathcal{V}, \Psi)$ is $S b T_{k}$ for $k=0,1,2$.

Proposition 9. Let $g:(\mathcal{U}, \tau) \rightarrow(\mathcal{V}, \Psi)$ be an injective supra $b^{\star}$-continuous mapping. If $(\mathcal{U}, \tau)$ is $S b T_{k}$, then $(\mathcal{V}, \Psi)$ is $S b T_{k}$ for $k=0,1,2$.

Proposition 10. Let $g:(\mathcal{U}, \tau) \rightarrow(\mathcal{V}, \Psi)$ be a bijective supra $b^{\star}$-open mapping. If $(\mathcal{U}, \tau)$ is $S b T_{k}$, then $(\mathcal{V}, \Psi)$ is $S b T_{k}$ for $k=0,1,2$.

Proposition 11. Let $g:(\mathcal{U}, \tau) \rightarrow(\mathcal{V}, \Psi)$ be a supra $b^{\star}$-homeomorphism mapping. Then $(\mathcal{U}, \tau)$ is $\operatorname{SbT}_{k}$ iff $(\mathcal{V}, \Psi)$ is $S b T_{k}$ for $k=0,1,2,3,4$.

Recall that: $(O \times P) \bigcup(C \times D) \subseteq(O \bigcup C) \times(P \bigcup D)$ for every $O, C \subseteq \mathcal{U}$ and $P, D \subseteq Y$.

Theorem 13. The product of two supra b-open sets $O$ and $P$ is supra b-open.

Proof. For two supra $b$-open sets $O$ and $P$, we have $O \subseteq \operatorname{int}(\operatorname{cl}(O)) \cup \operatorname{cl}(\operatorname{int}(O))$ and $P \subseteq \operatorname{int}(\operatorname{cl}(\operatorname{int}(P))) \bigcup \operatorname{int}(\operatorname{cl}(\operatorname{int}(P)))$. Therefore

$O \times P \subseteq[\operatorname{int}(\operatorname{cl}(O)) \bigcup \operatorname{cl}(\operatorname{int}(O))] \times[\operatorname{int}(\operatorname{cl}(P)) \bigcup \operatorname{cl}(\operatorname{int}(P))]$

$\subseteq[\operatorname{int}(\operatorname{cl}(O)) \times \operatorname{int}(\operatorname{cl}(P))] \bigcup[\operatorname{cl}(\operatorname{int}(O)) \times \operatorname{cl}(\operatorname{int}(P))]$

$=[\operatorname{int}(\operatorname{cl}(O \times P))] \bigcup[\operatorname{cl}(\operatorname{int}(O \times P))]$.

Thus, $O \times P$ is supra $b$-open.

Theorem 14. The finite product of $S b T_{k}$-spaces is $S b T_{k}$ for $k=0,1,2$.

Proof. We give a proof for two $\operatorname{STSs}(\mathcal{U}, \Omega)$ and $(\mathcal{V}, \Psi)$ when $k=2$.

Consider $(\mathcal{U} \times \mathcal{V}, T)$ as the product of $\operatorname{STSs}(\mathcal{U}, \Omega)$ and $(\mathcal{V}, \Psi)$. Suppose that $\left(\xi_{1}, \zeta_{1}\right) \neq$ $\left(\xi_{2}, \zeta_{2}\right)$. Then, $\xi_{1} \neq \xi_{2}$ or $\zeta_{1} \neq \zeta_{2}$. Suppose, without loss of generality, that $\xi_{1} \neq \xi_{2}$. By hypothesis, there are disjoint supra $b$-open subsets $M$ and $N$ of $(\mathcal{U}, \Omega)$ respectively including $\xi_{1}$ and $\xi_{2}$. It follows from Theorem (13) that $M \times \mathcal{V}$ and $N \times \mathcal{V}$ are two supra $b$-open subsets of $(\mathcal{U} \times \mathcal{V}, T)$ respectively including $\left(\xi_{1}, \zeta_{1}\right)$ and $\left(\xi_{2}, \zeta_{2}\right)$ such that $(M \times \mathcal{V}) \widetilde{\bigcap}(N \times \mathcal{V})=\emptyset$. This proves that $(\mathcal{U} \times \mathcal{V}, T)$ is $S b T_{2}$.

Definition 16. Let $(\mathcal{U} \times \mathcal{V}, T)$ be the product of $\operatorname{STSs}(\mathcal{U}, \Omega)$ and $(\mathcal{V}, \Psi)$ such that $\mathcal{C}_{1}$ and $\mathcal{C}_{2}$ are respectively the classes of all supra b-open subsets of $(\mathcal{U}, \Omega)$ and $(\mathcal{V}, \Psi)$. Then $\beta=\left\{\Theta \times H: \Theta \in \mathcal{C}_{1}\right.$ and $\left.H \in \mathcal{C}_{2}\right\}$ forms a basis for an $S T \mathcal{C}$ on $\mathcal{U} \times \mathcal{V}$. We call $(\mathcal{U} \times \mathcal{V}, \mathcal{C})$ a $b$-finite product of supra spaces.

Lemma 1. Let $(\mathcal{U} \times \mathcal{V}, \mathcal{C})$ be the b-product of $S T S s(\mathcal{U}, \Omega)$ and $(\mathcal{V}, \Psi)$. If $E$ is a supra closed subset of $(\mathcal{U} \times \mathcal{V}, \mathcal{C})$, then $E=\bigcap_{k \in I}\left[\left(F_{k} \times \mathcal{V}\right) \cup\left(\mathcal{U} \times H_{k}\right)\right]$, where $F_{k}$ and $H_{k}$ are respectively supra b-closed subsets of $(\mathcal{U}, \Omega)$ and $(\mathcal{V}, \Psi)$.

Theorem 15. The b-finite product of $S b T_{k}$-spaces is $S T_{k}$ for $k=0,1,2,3$. 
Proof. We give a proof for two $\operatorname{STSs}(\mathcal{U}, \Omega)$ and $(\mathcal{V}, \Psi)$ when $k=3$.

Let $(\mathcal{U} \times \mathcal{V}, \mathcal{C})$ be the $b$-product supra space of $(\mathcal{U}, \Omega)$ and $(\mathcal{V}, \Psi)$. First, we demonstrate that $(\mathcal{U} \times \mathcal{V}, \mathcal{C})$ is $S T_{1}$. To do this, let $\left(\xi_{1}, \zeta_{1}\right) \neq\left(\xi_{2}, \zeta_{2}\right)$. Then, $\xi_{1} \neq \xi_{2}$ or $\zeta_{1} \neq \zeta_{2}$. Suppose, without loss of generality, that $\xi_{1} \neq \xi_{2}$. So that, there are supra $b$-open subsets $M$ and $N$ of $(\mathcal{U}, \Omega)$ respectively including $\xi_{1}$ and $\xi_{2}$. It comes from Definition(16) that $M \times \mathcal{V}$ and $N \times \mathcal{V}$ are two supra open subsets of $(\mathcal{U} \times \mathcal{V}, \mathcal{C})$ respectively including $\left(\xi_{1}, \zeta_{1}\right)$ and $\left(\xi_{2}, \zeta_{2}\right)$ such that $\left(\xi_{1}, \zeta_{1}\right) \notin N \times \mathcal{V}$ and $\left(\xi_{2}, \zeta_{2}\right) \notin M \times \mathcal{V}$. Thus, $(\mathcal{U} \times \mathcal{V}, \mathcal{C})$ is $S T_{1}$. Second, we demonstrate that $(\mathcal{U} \times \mathcal{V}, \mathcal{C})$ is supra regular. Suppose that $(x, y) \in \mathcal{U} \times \mathcal{V}$ and $E$ is a supra closed subset of $(\mathcal{U} \times \mathcal{V}, \mathcal{C})$ such that $(\xi, \zeta) \notin E=\bigcap_{k \in I}\left[\left(F_{k} \times \mathcal{V}\right) \cup\left(\mathcal{U} \times H_{k}\right)\right]$, where $F_{k}$ and $H_{k}$ are respectively supra $b$-closed subsets of $(\mathcal{U}, \Omega)$ and $(\mathcal{V}, \Psi)$. Then there is $j \in I$ such that $(\xi, \zeta) \notin\left[\left(F_{j} \times \mathcal{V}\right) \bigcup\left(\mathcal{U} \times H_{j}\right)\right]$. This means that $\xi \notin F_{j}$ and $\zeta \notin H_{j}$. Since $(\mathcal{U}, \Omega)$ and $(\mathcal{V}, \Psi)$ are supra $b$ regular, there are disjoint supra $b$-open subsets $O$ and $P$ of $(\mathcal{U}, \Omega)$ respectively including $\xi$ and $F_{j}$, and there are disjoint supra $b$-open subsets $M$ and $N$ of $(\mathcal{V}, \Psi)$ respectively including $\zeta$ and $H_{j}$. Now, $O \times M$ and $[(P \times \mathcal{V}) \bigcup(\mathcal{U} \times N)]$ are two supra open subsets of $(\mathcal{U} \times \mathcal{V}, \mathcal{C})$ respectively including $(\xi, \zeta)$ and $\left[\left(F_{j} \times \mathcal{V}\right) \bigcup\left(\mathcal{U} \times H_{j}\right)\right]$. Obviously, $E \subseteq\left[\left(F_{j} \times \mathcal{V}\right) \bigcup\left(\mathcal{U} \times H_{j}\right)\right]$ and $(O \times M) \bigcap[(P \times \mathcal{V}) \bigcup(\mathcal{U} \times N)]=\emptyset$. Hence, $(\mathcal{U} \times \mathcal{V}, T)$ is supra regular.

\section{Conclusion}

Supra topology is one of the famous extensions of topological spaces. We have devoted this article to present novel types of limit points and separation axioms in the frame of supra topology. We have formulated these types using supra $b$-open sets. We have scrutinized their basic features and revealed some relationships between them. To validate the introduced findings, we have displayed some illustrative examples. To complete this path of study, we plan to investigate the following topics:

(i) Formulate other kinds of regular and normal spaces.

(ii) Explore strong types of separation axioms induced from supra $b$-open set like $S b T_{k^{-}}$ spaces for $\left(k=\frac{1}{2}, 2 \frac{1}{2}, 3 \frac{1}{2}\right)$.

\section{Conflict of interest}

The authors declare that there is no conflict of interest regarding the publication of this paper.

\section{Acknowledgements}

This publication was supported by the Deanship of scientific research at Prince Sattam bin Abdulaziz University, Alkharj, Saudi Arabia. 


\section{References}

[1] A M Al-Odhari. On infra topological spaces. International Journal of Mathematical Archive, 6(11):179-184, 2015.

[2] T M Al-shami. Some results related to supra topological spaces. Journal of Advanced Studies in Topology, 4(7):283-294, 2016.

[3] T M Al-shami. On supra semi open sets and some applications on topological spaces. Journal of Advanced Studies in Topology, 8(2):144-153, 2017.

[4] T M Al-shami. Somewhere dense sets and $S T_{1}$-spaces. Punjab University Journal of Mathematics, 49(2):101-111, 2017.

[5] T M Al-shami. Utilizing supra $\alpha$-open sets to generate new types of supra compact and supra lindelöf spaces. Facta Universitatis, Series: Mathematics and Informatics, 32(1):151-162, 2017.

[6] T M Al-shami. Supra semi-compactness via supra topological spaces. Journal of Taibah University for Science, 12(3):338-343, 2018.

[7] T M Al-shami. Paracompactness on supra topological spaces. Journal of Linear and Topological Algebra, 9(2):1-7, 2020.

[8] T M Al-shami. Complete hausdorffness and complete regularity on supra topological spaces. Journal of Applied Mathematics, Volume 2021, Article ID 5517702:11 pages, 2021.

[9] T M Al-shami, E A Abo-Tabl, and B A Asaad. Investigation of limit points and separation axioms using supra $\beta$-open sets. Missouri Journal of Mathematical Science, 32(2):171-187, 2020.

[10] T M Al-shami, E A Abo-Tabl, B A Asaad, and M A Arahet. Limit points and separation axioms with respect to supra semi-open sets. European Journal of Pure and Applied Mathematics, 13(3):427-443, 2020.

[11] T M Al-shami, B A Asaad, , and M K EL-Bably. Weak types of limit points and separation axioms on supra topological spaces. Advances in Mathematics: Scientific Journal, 9(10):8017-8036, 2020.

[12] T M Al-shami, B A Asaad, and M A El-Gayar. Various types of supra pre-compact and supra pre-lindelöf spaces. Missouri Journal of Mathematical Science, 32(1):1-20, 2020 .

[13] T M Al-shami and M E El-Shafei. On supra soft topological ordered spaces. Arab Journal of Basic and Applied Sciences, 26(1):433-445, 2019. 
[14] T M Al-shami and M E El-Shafei. Two types of separation axioms on supra soft topological spaces. Demonstratio Mathematica, 52(1):147-165, 2019.

[15] T M Al-shami, M Al Shumrani, and C Özel. Another form of supra ordered separation axioms. Journal of Mathematical Extension, 15(1):105-125, 2021.

[16] T M Al-shami and T Noiri. More notions and mappings via somewhere dense sets. Afrika Matematika, 30(7):1011-1024, 2019.

[17] Á Császár. Generalized topology, generalized continuity. Acta Mathematica Hungarica, 96:351-357, 2002.

[18] R Devi, S Sampathkumar, and M Caldas. On $\alpha$-open sets and $s \alpha$-continuous maps. General Mathematics, 16:77-84, 2008.

[19] M E El-Shafei, M Abo-Elhamayel, and T M Al-shami. On supra $R$-open sets and some applications on topological spaces. Journal of Progressive Research in Mathematics, 8(2):1237-1248, 2016.

[20] M E El-Shafei, A H Zakari, and T M Al-shami. Some applications of supra preopen sets. Journal of Mathematics, Volume 2020, Article ID 9634206:11 pages, 2020.

[21] S Jafari and S Tahiliani. Supra $\beta$-open sets and supra $\beta$-continuity on topological spaces. Annales Univ. Sci. Budapest., 56:1-9, 2013.

[22] R M Latif. Supra-r-compactness and supra-r-connectedness. International Journal of Recent Trends in Engineering 63 Research, 4(1):2455-1457, 2018.

[23] H Maki, J Umehara, and T Noiri. Every topological space is pret $t_{\frac{1}{2}}$. Mem. Fac. Sci. Kochi. Univ. Ser. A Math., 17:33-42, 1996.

[24] A S Mashhour, A A Allam, F S Mahmoud, and F H Kheder. On supra topological spaces. Indian Journal of Pure and Applied Mathematics, 14(4):502-510, 1983.

[25] J M Mustafa. Supra b-compact and supra b-lindelof spaces. Journal of Mathematics and Applictions, 36:79-83, 2013.

[26] J M Mustafa and H A Qoqazeh. Supra d-sets and associated separation axioms. International Journal of Pure and Applied Mathematics, 80(5):657-663, 2012.

[27] O R Sayed. Supra pre-open sets and supra pre-continuous on topological spaces. Series Mathematics and Information, 20(2):79-88, 2010.

[28] O R Sayed. Supra $\beta$-connectedness on topological spaces. Proceedings of the Pakistan Academy of Sciences, 49(1):19-23, 2012.

[29] O R Sayed and T Noiri. On supra b-open sets and supra b-continuity on topological spaces. European Journal of Pure and Applied Mathematics, 3:295-302, 2010. 